\title{
What Can We Learn about Aging and COVID-19 by Studying Mortality?
}

\author{
L. A. Gavrilov ${ }^{1,2, a *}$ and N. S. Gavrilova ${ }^{1,2}$ \\ ${ }^{1}$ Academic Research Centers, NORC at the University of Chicago, 60637 Chicago, IL, USA \\ ${ }^{2}$ Institute for Demographic Research, Federal Center of Theoretical and Applied Sociology, \\ Russian Academy of Sciences, 109028 Moscow, Russia \\ ${ }^{a}$ e-mail: gavrilov@longevity-science.org
}

Received July, 09, 2020

Revised August, 22, 2020

Accepted August, 23, 2020

\begin{abstract}
Promising ideas and directions for further research into biology of aging are discussed using analysis of the agerelated kinetics of organisms' mortality. It is shown that the traditional evolutionary theory explaining aging by decreasing force of natural selection with age is not consistent with the data on age-related mortality kinetics. The hypothesis of multistage destruction of organisms with age, including the rate-limiting stage of transition to a state of non-specific vulnerability ("non-survivor"), is discussed. It is found that the effect of the COVID-19 coronavirus infection on mortality is not additive (as it was the case with the Spanish flu epidemic in 1918), but multiplicative (proportional) for ages over 65 years.
\end{abstract}

DOI: $10.1134 / \mathrm{S} 0006297920120032$

Keywords: aging, mortality, COVID-19, non-survivor, mortality kinetics

\section{INTRODUCTION}

Analysis of the kinetics of biological processes is widely used in biochemistry (kinetics of enzymatic catalysis) and other areas of biology, including biology of aging and lifespan [1]. The aim of this paper is to discuss promising ideas and directions for further research in the biology of aging, using analysis of the age-related kinetics of organism mortality.

Fundamental biological theories of aging can be tested using the data on mortality and longevity. For example, traditional evolutionary theory explains aging by the decreasing force of natural selection with age. According to the mutation accumulation theory proposed by Peter Medawar, the equilibrium frequency of harmful mutations is higher for mutations operating at older ages (MOA), as the selection against MOA is weaker and the balance between formation of the new mutations and their selection-out shifts towards higher levels of MOA [2]. One of the testable predictions of this theory is prediction of a fundamental change in the age-specific dynamics of mortality at very old post-reproductive ages, when the force of natural selection becomes negligible and there is no possibility for its further reduction. For

* To whom correspondence should be addressed. example, a prediction could be made that the dynamics of mortality at reproductive age (20-40 years in humans) should fundamentally differ from the dynamics of mortality at extreme post-reproductive ages ( $90-105$ years).

\section{TESTING PREDICTIONS OF THE EVOLUTIONARY THEORY OF AGING}

We tested this hypothesis using the U. S. Social Security Administration Death Master File (DMF) data. It was found in the study of ten single-year extinct birth cohorts born in 1886-1895 that the rate of mortality growth with age at post-reproductive old age (up to 105 years) is exactly the same as at younger reproductive ages and is consistent with the Gompertz-Makeham law [3]. This conclusion was further confirmed by studies of mortality in 22 single-year birth cohorts of the US men and women born in 1890-1900 (data from the Human Mortality Database), and by analysis of the mortality trajectories in 8 cohorts of the laboratory mouse and 10 cohorts of the laboratory rats [4].

Figure 1 illustrates this finding. It can be seen that the mortality rate of women in the United States at ages 100-105 years continues to increase exponentially, according to the trajectory started at an earlier age. 


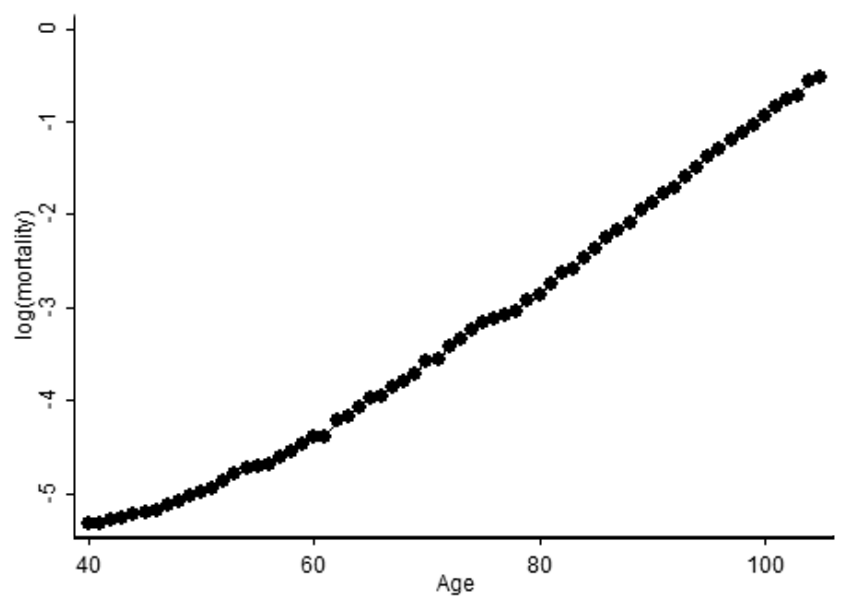

Fig. 1. Logarithm of mortality force for US women born in 1898 as a function of age. Data source: Human Mortality Database.

This observation represents a challenge to many theories of aging, including the evolutionary theory that explains aging by declining force of natural selection with age. New ideas are needed to explain why exactly the same exponential dynamics of mortality growth is observed not only at the reproductive age, but also at the very old post-reproductive age (up to 105 years) when the force of natural selection becomes close to zero (and there is no room for its further reduction).

\section{AGE-RELATED AND HISTORICAL DYNAMICS OF MORTALITY}

It should be noted that the dependence of human mortality on age $x$ after age 30 years is well described by the well-known Gompertz-Makeham formula with three non-negative parameters $\mathrm{A}, \mathrm{R}$, and $\alpha[1]$ :

$$
\mu(\mathrm{x})=\mathrm{A}+\mathrm{R} \exp (\alpha \mathrm{x}) .
$$

Here, the background mortality (parameter A) does not depend on age and reflects mortality from external (exogenous) causes of death, while the age-related mortality (Gompertz term) increases exponentially with age and is associated with aging of the organism. To some extent these two components correspond to exogenous (external) and endogenous causes of death. [1,5]. Until the 1950s, the decline in human mortality was mainly due to the decline in background mortality, while the agerelated mortality remained relatively stable [1] (see also Fig. 2: the mortality curves for calendar years 1925 and 1955 are almost the same for ages over 80). This decline in mortality was achieved mainly due to the successful control of infectious diseases. In the 1960s, the background mortality decreased to an extremely low level close to zero and this reserve for the further mortality reduction was exhausted. As a result, most developed countries experienced a brief stagnation period in life expectancy growth in the 1960s [6,7].

In the second half of the 20th century, medicine was able to influence the age-related component of mortality and it began to decline mainly due to the decrease in mortality from cardiovascular diseases [7]. However, these successes were accompanied in some cases by the increase in mortality from other causes (e.g., Alzheimer's disease) $[8,9]$. Thanks to advances in medicine in the fight against chronic diseases of old age, most developed countries have been able to achieve reductions in the age-related mortality after the mid-1970s (see Fig. 2, mortality curves at older ages in calendar years 1955 and 2010). This decline in the age-related component of mortality in developed countries is still observed today, although some countries have experienced another stagnation in mortality decline (e.g., the United States). It can be seen from Fig. 2 that the decline in mortality over the period from 1955 to 2010 looks like a parallel shift of mortality down in semi-logarithmic coordinates. Thus, in modeling the reduction of mortality over the recent years, it is possible to use a model of proportional risks with a constant multiplier $a$ :

$$
m_{x+t}=a m_{x}
$$

where $0<\mathrm{a}<1 ; m_{x+t}-$ mortality at age $x$, after time period $t$.

This property of proportional mortality decline is currently used in mortality forecasting [10]. Such change in mortality can be interpreted as a shift of age back in the exponential function by the same number of years in all age groups, or as a formal "rejuvenation" occurring in all age groups equally. Indeed, a recent study found that the functional status and aging biomarkers of older Japanese

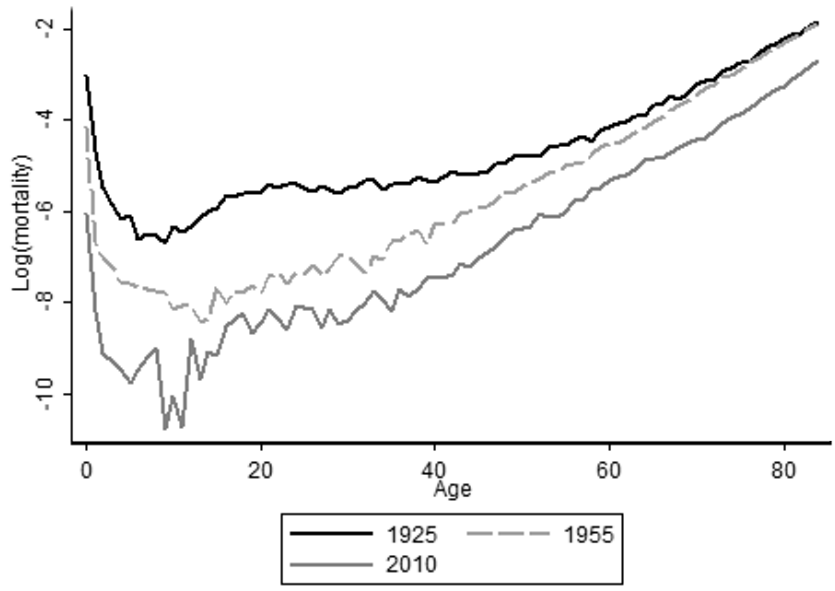

Fig. 2. Logarithm of mortality as a function of age for Swedish women in 1925, 1955 and 2010. Data source: Human Mortality Database (HMD). 
residents in 2017 were consistent with those of younger ages (compared with the biomarkers measured ten years ago for people of the same age) [11]. A similar result was obtained by examining the functional status of Europeans. The cognitive and physical functioning indicators measured in year 2013 formally corresponded to younger ages compared to the cohorts of the same calendar age in 2004 [12]. Thus, in recent years there has been a gradual functional "rejuvenation" of cohorts of people of the same calendar age. However, the mortality rate of centenarians has not practically changed for the last 80 years [13]. Thus, the model of proportional change of mortality over time appears inapplicable to extremely old ages.

\section{RELIABILITY THEORY AND “NON-SURVIVOR” HYPOTHESIS}

Discussing the age-related dynamics of mortality, we should consider the following relevant question in the study of aging: how is it possible for different diseases and causes of death to "negotiate" with each other in order to produce a simple exponential function for mortality from all causes of death combined (given that contribution of the different causes of death to total mortality varies greatly with age)? Linked to this question is the traditional approach to life extension, based on combating individual causes of death. Indeed, it is well known, from which causes of death the mortality decreased in the first half of the 20th century. These are primarily pneumonia, influenza, tuberculosis, enteritis and other infectious diseases [14]. It is also known that mortality from each of these causes changes with age. Therefore, their elimination should inevitably change the age dynamics of total mortality and the size of its age-related component. However, mortality increases with age according to the fairly simple Gompertz formula (the Makeham term is close to zero in recent decades and has little effect on mortality dynamics). The only way to resolve this contradiction is to admit that the causes of death are not independent of each other, but are coordinated so that the age-related component of mortality increases exponentially with age, despite a dramatic change in the structure of causes of death. However, then the following question arises: how do the causes of death "agree" with each other so that the age-related component of mortality grows with age in accordance with a fairly simple Gompertz law?

The above facts can be explained by using the hypothesis of limited organism's reliability. According to this hypothesis, an organism is a multi-redundant system with high, but not infinitely high reliability [1, 15]. Therefore, there is always some probability that the interference in the work of individual elements of the organism will coincide randomly in time and the organism will move into a state of non-specific vulnerability. Such failure causes a whole cascade of dependent failures of other systems in the organism, so there are many observed causes of death. Figure 3 shows the simplest scheme illustrating the idea of this hypothesis. According to this scheme, an organism in a normal state can die only in extreme situations, certainly lethal for any, even the healthiest organism (corresponding to the background component of mortality, which in the developed countries is already close to zero). In addition, as a result of the failure of one of the body systems, it may also pass into a state of nonspecific vulnerability, which is called "non-survivor" [1]. It should be noted that this state has a quite clear biological meaning. For example, failures of immune system, the frequency of which sharply increases with age, create a nonspecific vulnerability to the widest range of diseases, both endogenous and exogenous [16, 17]. Having fallen into a state of nonspecific vulnerability, an organism quickly dies from any of the first causes it has caught. This concept to a certain extent echoes the new concept of phenoptosis, when an organism is eliminated from the population as a result of multiple systems failure [18].

As can be seen from this scheme, the age-related component of mortality is determined by the rate of the first limiting stage of the organism's transition from a normal state to a state of non-specific vulnerability ("non-survivor"). This means that the age-related component of mortality is not summed-up of individual causes of death but, on the contrary, is being distributed between them. In other words, the rate of the first limiting stage determines the value of "death quota", which is then distributed among its various particular manifestations, called "causes" of death. The proposed scheme allows explaining why elimination of the separate agedependent causes of death is not always capable of changing the size of the age component of mortality. In fact, any reduction of the death rate of organisms, being in a state of non-specific vulnerability, inevitably leads to the increase of share of the organisms being in this state, and to restoration of the former mortality rate due to increase of mortality from other causes. However, when the rate of the rate-limiting stage decreases, the transition to a state of nonspecific vulnerability is slowed down and the total mortality rate decreases.

The hypothesis of limited organism's reliability explains the phenomenon of historical stability of the age-related component of mortality before the early 1950s, as well as the facts of "independent" behavior of the total mortality in relation to its components. Moreover, this hypothesis makes it possible to justify the Gompertz-Makehan formula using such simple notions of the nature of aging as reduction of reserves of organism systems with age [1]. Therefore, the idea of limited reliability of the organism is sufficiently well-founded and natural to be used as a working hypothesis in determining the ways and prospects for extension of human life.

This hypothesis argues that the problem of human lifespan extension is not reduced to fighting individual 




Fig. 3. Simplified scheme illustrating hypothesis of intermediate state of non-specific vulnerability ("non-survivor").

causes of death. Moreover, the hypothesis of limited reliability predicts that reduction of mortality from individual causes of death will only lead to a significant reduction of total mortality when the initial stage of organism's destruction (transition to a state of non-specific vulnerability) ceases to be a limiting stage of the whole process. Apparently, the future belongs to another strategy based on explaining the mechanisms of organism's reliability providing nonspecific resistance to a wide range of damaging factors. If successful in this direction, we can expect simultaneous reduction in mortality from a wide variety of diseases. These ideas are conceptually close to the currently developing direction in gerontology, which is called geroscience [19]. This direction is based on the well-



Fig. 4. Trends in logarithm of mortality for Spanish women in year 2020 for three age groups, 65-74, 75-84 and 85+ years, by week. Data source: Human Mortality Database (HMD). known idea that in order to increase lifespan and healthy longevity in particular, it is necessary to move from combating specific diseases of old age to slowing down the pathological processes leading to aging (e.g., reduction of systemic sterile inflammation). Apparently, further success in gerontology should be expected in the development of this particular direction of research.

\section{MORTALITY DYNAMICS IN THE TIME OF COVID-19}

Despite the successful fight against infectious diseases, the current coronavirus epidemic shows that the



Fig. 5. Logarithm of mortality as a function of age before coronavirus epidemic (5th week of 2020, clear markers) and during the coronavirus epidemic (14th week of 2020, black markers) for Spanish men and women. Data source: Human Mortality Database. 
final victory in this fight is far from over. Scientists have already noticed that COVID-19 affects mainly the old people [20]. In this respect, it is useful to check how COVID-19 affects the age profile of mortality and aging rate. Infectious diseases have tended to increase background mortality in the past, i.e., mortality in all age groups received an additional and approximately the same component for all ages $a$ :

$$
m_{x+t}=A+a+m_{x},
$$

where $A$ is background mortality, $a$ - additional component of background mortality as a result of epidemic, $m_{x}-$ age-related mortality at age $x$ before epidemic. This was the mechanism by which the mortality rate increased during the Spanish flu pandemic in 1918 [21]. Because of this, mortality at young and middle ages increased by a factor of about 3.3, while at older ages it increased by a factor of only 1.3 (calculated from HMD data for Italian women). This was the result of an additive rather than a multiplicative mechanism of increasing mortality during the Spanish flu pandemic [21].

Now let's look at the current COVID-19 pandemic. The Human Mortality Database contains information on weekly deaths in a number of countries [22]. Data for Spain provide an opportunity to compare mortality at the peak of the epidemic with mortality before the epidemic. Figure 4 shows trends in the logarithm of mortality for Spanish women in year 2020 for three age groups, 65-74, $75-84$, and $85+$ years. It may be noted that, for all three age groups, the peak of mortality occurred around the 14 th week of 2020.

The effect of coronavirus infection on the age-specific mortality can be analyzed by comparing mortality at the 14th week (peak of the epidemic) with mortality at the 5 th week (before the epidemic). The age-specific mortality rates in HMD are given for wide age intervals but nevertheless allow estimating the change in the age-specific dynamics of mortality before and during the COVID-19 epidemic for ages above 65 years.

Figure 5 shows the age profile of the logarithm of mortality in Spanish elderly population before and during the epidemic for both men and women. It can be seen that there is a fairly clear upward parallel shift in mortality for both women and men. Thus, a model of proportional risks with a constant multiplier $a$ can be applied to mortality description during the COVID-19 epidemic:

$$
m_{x+t}=a m_{x}, \text { where } a>1 .
$$

The mortality rate of the elderly population of Spain (over 65 years of age) at the time of the epidemic was proportionally increased at older ages by a factor of 1.9 for men and 1.7 for women (see Fig. 5). It is interesting to note that, at younger ages (under 50), the COVID-19 pandemic had little or no effect on the total mortality rate in that country (as found in our analysis of more detailed data on five-year age groups in the Human Mortality Database).

Unlike the model of proportional reduction of mortality in human history discussed earlier, this is not a formal "rejuvenation", but on the contrary a shock "aging" of the population during the epidemic. In the framework of the hypothesis under discussion, it is possible to assume that the transition to a state of non-specific vulnerability ("non-survivor") occurs more rapidly under the action of coronavirus infection, or that this infection "eliminates" non-survivors more rapidly ("harvesting effect"). The multiplicative effect of the increase in mortality during the COVID-19 epidemic is a significant difference between this epidemic and the 1918 Spanish flu pandemic [21]. This mechanism also explains the predominant killing of the older population compared to the Spanish flu in 1918. As in the case of Spanish flu epidemic, the actuarial rate of aging (relative growth rate of mortality with age) does not change at older ages (65+ years). However, the so-called "compensation effect of mortality" (reduction of relative differences in mortality with age [1] is clearly observed when comparing mortality of men and women both before and during the epidemic (see Fig. 5, convergence of male and female death rates at older ages).

Thus, the analysis of age-specific mortality dynamics allows researchers to study possible mechanisms of its change during aging and stressful infectious loads.

Funding. This work was partially financially supported by the National Institutes of Health (project no. NIH R21AG054849).

Acknowledgments. The authors express their gratitude to the participants of the international conference "Homo Sapiens Liberatus" held at the Moscow State University (Moscow, February 2020) and Academician Vladimir Petrovich Skulachev for useful discussion of the results of this study [23].

Ethics declarations. The authors declare no conflict of interest in financial or any other sphere. This article does not contain any studies with human participants or animals performed by any of the authors.

\section{REFERENCES}

1. Gavrilov, L. A., and Gavrilova, N. S. (1991) The Biology of Life Span: A Quantitative Approach, Harwood Academic Publisher, New York

2. Gavrilov, L. A., and Gavrilova, N. S. (2002) Evolutionary theories of aging and longevity, ScientificWorldJournal, 2, 339-356, doi: $10.1100 /$ tsw.2002.96.

3. Gavrilov, L. A., and Gavrilova, N. S. (2011) Mortality measurement at advanced ages: a study of the Social Security Administration Death Master File, N. Amer. 
Actuarial J., 15, 432-447, doi: 10.1080/10920277.2011. 10597629.

4. Gavrilova, N. S., and Gavrilov, L. A. (2015) Biodemography of old-age mortality in humans and rodents, J. Gerontol. Ser. A Biol. Sci. Med. Sci., 70, 1-9, doi: $10.1093 /$ gerona/glu009.

5. Carnes, B. A., Holden, L. R., Olshansky, S. J., Witten, T. M., and Siegel, J. S. (2006) Mortality partitions and their relevance to research on senescence, Biogerontology, 7, 183-198, doi: 10.1007/s10522-006-9020-3.

6. Gavrilov, L. A., Gavrilova, N. S., and Nosov, V. N. (1983) Human life span stopped increasing: why? Gerontology, 29, 176-180, doi: 10.1159/000213111.

7. Ouellette, N., Barbieri, M., and Wilmoth, J. R. (2014) Period-based mortality change: turning points in trends since 1950, Popul. Dev. Rev., 40, 77-106, doi: 10.1111/ j.1728-4457.2014.00651.x.

8. Mesle, F., and Vallin, J. (2006) Diverging trends in female old-age mortality: The United States and the Netherlands versus France and Japan, Popul. Dev. Rev., 32, 123-145, doi: 10.1111/j.1728-4457.2006.00108.x.

9. Mesle, F., and Vallin, J. (2011) Historical trends in mortality, in International Handbook of Adult Mortality (Rogers, R., and Crimmins, E., eds.) Springer, New York, pp. 9-47

10. Lee, R. (2013) The Lee-Carter method for forecasting mortality, with various extensions and applications, N. Amer. Actuarial J., 4, 80-91, doi: 10.1080/10920277. 2000.10595882.

11. Suzuki, T., Nishita, Y., Jeong, S., Shimada, H., Otsuka, R., et al. (2020) Are Japanese older adults rejuvenating? Changes in health-related measures among older community dwellers in the last decade, Rejuv. Res., doi: 10.1089/ rej.2019.2229.

12. Ahrenfeldt, L., Lindahl-Jacobsen, R., Rizzi, S., Thinggaard, M., Christensen, K., and Vaupel, J. (2018) Comparison of cognitive and physical functioning of Europeans in 2004-05 and 2013, Int. J. Epidemiol., 47, 1518-1528, doi: 10.1093/ije/dyy094.

13. Gavrilov, L. A., Gavrilova, N. S., and Krut'ko, V. N. (2017) The future of human longevity, Gerontology, 63, 524-526, doi: 510.1159/000477965.
14. Bourgeois-Pichat, J. (1979) Future outlook for mortality decline in the world, in Prospects of Population: Methodology and Assumptions, United Nations, NY, pp. 227-266.

15. Gavrilov, L. A., and Gavrilova, N. S. (2006) Reliability theory of aging and longevity, in Handbook of the Biology of Aging (Masoro, E. J., and Austad, S. N., eds.) 6 Edn., Academic Press, San Diego, pp. 3-42.

16. Franceschi, C., Garagnani, P., Parini, P., Giuliani, C., and Santoro, A. (2018) Inflammaging: a new immune-metabolic viewpoint for age-related diseases, Nat. Rev. Endocrinol., 14, 576-590, doi: 10.1038/s41574-018-0059-4.

17. Naumova, E., Ivanova, M., and Pawelec, G. (2011) Immunogenetics of ageing, Int. J. Immunogenet., 14, 576590, doi: 10.1111/j.1744-313X.2011.01022.x.

18. Skulachev, V. P., Shilovsky, G. A., Putyatina, T. S., Popov, N. A., Markov, A. V., Skulachev, M. V., and Sadovnichii, V. A. (2020) Perspectives of Homo sapiens lifespan extension: focus on external or internal resources? Aging, 12, 5566-5584, doi: 10.18632/aging. 102981.

19. Burch, J., Augustine, A., Frieden, L., Hadley, E., Howcroft, T., et al. (2014) Advances in geroscience: impact on healthspan and chronic disease, J. Gerontol. Ser. A Biol. Sci. Med. Sci., 69, S1-S3, doi: 10.1093/gerona/glu041.

20. Blagosklonny, M. (2020) From causes of aging to death from COVID-19, Aging, 12, 10004-10021, doi: 10.18632/ aging. 103493.

21. Gavrilova, N. S., and Gavrilov, L. A. (2020) Patterns of mortality during pandemic: an example of Spanish flu pandemic of 1918, Population Economics, 4, 56-64, doi: 10.3897/popecon.4.e53492.

22. Human Mortality Database. University of California, Berkeley (USA), and Max Planck Institute for Demographic Research (Germany). Available at www.mortality. org or www.humanmortality.de (retrieved on 6.5.2020)

23. Gavrilov, L. A., and Gavrilova, N. S. (2020) Matters of life and death: what can we learn about aging from mortality and longevity studies? In Homo Sapience Liberatus. Proceedings of the 3rd International Conference in Celebration of the 85th Birthday of Professor V. P. Skulachev. Abstract Book, Torus Press, Moscow, pp. 27-28, doi: 10.30826/HomoSapiens-2020-16. 